\title{
Natura@economía
}

ISSN 2226-9479 (Versión electrónica)

Website: http://revistas.lamolina.edu.pe/index.php/neu

ARTÍCULO ORIGINAL

\section{Equilibrio en el largo plazo y causalidad entre exportaciones mineras metálicas, producción industrial y crecimiento económico en el Perú 1994-2016}

Long-term balance and causality between metal mining exports, industrial production and economic growth in Peru 1994-2016

Anthony Paul Luna Farias ${ }^{1 *}$; Hugo Ibrahim Luna Astorga ${ }^{1}$

${ }^{1}$ Facultad de Economía y Planificación, Universidad Nacional Agraria La Molina, Lima, Perú. E-mail: 20111137@ lamolina.edu.pe; hugoluna@lamolina.edu.pe

Recepción: 10/06/2020; Aceptación: 15/12/2020

\begin{abstract}
La presente investigación ha tenido como objetivo analizar las exportaciones mineras metálicas y la producción industrial para determinar cuál de ellos tuvo mayor incidencia sobre el crecimiento económico en el Perú en el periodo 1994-2016. Basándose en un Modelo de Vectores Autorregresivos (VAR), se utilizó las pruebas de Cointegración de Johansen y Causalidad de Granger, tomando como variables al Producto Bruto Interno (PBI), Valores FOB de las exportaciones mineras metálicas (XMIN) y el Producto Bruto Interno manufacturero (PBIMAN). Los resultados sugieren que (1) las exportaciones mineras metálicas mantuvieron una relación de equilibrio en el largo plazo con el crecimiento económico del Perú y con la producción manufacturera. (2) Existe una relación de causalidad unidireccional desde las exportaciones de minerales metálicos y la producción industrial hacia el crecimiento económico del Perú. Asimismo, existe una relación de causalidad bidireccional entre el sector industrial y las exportaciones de minerales metálicos. Finalmente, se concluye que el efecto determinante para el crecimiento económico peruano en el periodo 1994-2016 ha sido el de las exportaciones mineras metálicas con una velocidad de ajuste de $24,7 \%$ hacia el equilibrio en el largo plazo.
\end{abstract}

Resumen

Palabras clave: Crecimiento económico; exportaciones; manufactura; vectores autorregresivos; cointegración; causalidad.

Forma de citar el artículo: Luna, A.; Luna, H. 2020. Equilibrio en el largo plazo y causalidad entre exportaciones mineras metálicas, producción industrial y crecimiento económico en el Perú 1994-2016. Natura@economía 5(2):72-87 (2020). http://dx.doi.org/10.21704/ne.v5i2.1608

DOI: http://dx.doi.org/10.21704/ne.v5i2.1608

* Autor de correspondencia: Luna, H. Email: hugoluna@lamolina.edu.pe

(C) Facultad de Economía y Planificación, Universidad Nacional Agraria La Molina, Lima, Perú. 


\begin{abstract}
The objective of this research has been to analyze metal mining exports and industrial production to determine which of them had the greatest impact on economic growth in Peru in the period of 1994-2016. Based on a Vector Autoregressive model (VAR), the Johansen's Cointegration and Granger's causality tests were used, taking as variables the Gross Domestic Product (PBI), FOB Values of metallic mining exports (XMIN) and the Gross Domestic Product Manufacturing (PBIMAN). The results suggest that (1) the metallic mining exports maintained an equilibrium relation in the long term with the economic growth of Peru and with the manufacturing production. (2) There is a unidirectional causality relationship from exports of metallic minerals and industrial production to the economic growth of Peru. Furthermore, there is a bidirectional causality relation between the industrial sector and exports of metallic minerals. Finally, it's concluded that the determining effect for the peruvian economic growth in the period of 1994-2016 has been the metallic mining exports at a rate of $24,7 \%$ adjustment towards equilibrium in the long run.
\end{abstract}

Keywords: Economic growth; exports; manufacture; vector autoregressive; cointegration; causality.

\section{Introducción}

El Perú es el país con mayor atractivo para la inversión minera en la región. Debido a la concentración de inversiones en el sector minero y a los altos precios internacionales, los minerales metálicos han llegado a representar el 59\% de las exportaciones totales del país al 2016, teniendo un impacto directo sobre el producto bruto interno (Instituto Peruano de Economía, 2017).

Las exportaciones mineras metálicas son de gran importancia para aumentar la producción manufacturera, ya que, a través de ingresos en divisas, suministra al sector industrial de bienes de capital y actualiza su tecnología existente (Instituto Peruano de Economía, 2017).

$\mathrm{Si}$ bien es cierto que las exportaciones mineras metálicas generan un efecto directo sobre el PBI, también ocasionan un efecto indirecto que se ve reflejado en el aumento de la producción manufacturera, el cual genera externalidades positivas que impulsan el crecimiento económico. En ese sentido, surge la duda sobre cuál ha sido el efecto determinante para lograr el crecimiento económico en el Perú.
En el ámbito internacional, Sahoo et al. (2014), Ee (2016) y Shafiullah et al. (2016) realizaron investigaciones en India, África subsahariana y Australia respectivamente, encontrando que las exportaciones mineras fueron determinantes para el crecimiento económico de esos países. Por otro lado, Hausmann et al. (2007) y Tang et al. (2015) sostienen que los países se deben concentrar en producción manufacturera para ser exportada, ya que los productos de exportación que cuentan con una alta tecnología se benefician de externalidades positivas, impulsando el crecimiento económico.

En el ámbito nacional, tanto Bobadilla (2016) como Díaz y Torres (2016), sostienen que a partir de los 90's el efecto de las exportaciones mineras metálicas ha tenido un impacto positivo sobre el crecimiento económico peruano, manteniéndose una relación de equilibrio en el largo plazo. Por el contrario, Jiménez (2017) señala que la manufactura ha sido el factor determinante para el crecimiento económico que ha tenido el Perú desde inicios la década de los noventa. Por lo tanto, no hay una concordancia en el sentido de cuál ha sido 
el factor determinante para el crecimiento económico en el Perú desde inicios de los noventa al 2015.

La inversión en la minería y el alto precio de los commodíties han permitido que aumenten las exportaciones mineras metálicas (Instituto Peruano de Economía, 2017), teniendo un impacto directo sobre el PBI y siendo determinante para el crecimiento económico (Sahoo et al., 2014; Bobadilla, 2016; Díaz y Torres, 2016; Ee, 2016 y Shafiullah et al., 2016). Asimismo, la gran cantidad de divisas obtenidas han permitido aumentar la producción manufacturera, la cual ha impulsado al crecimiento económico a través de las externalidades positivas que genera (Hausmann et al., 2007; Tang et al., 2015; Jiménez, 2017). Sin embargo, Ee (2016) y Alarco (2011) sostienen que la orientación de inversiones hacia el sector minero puede quitarle competitividad a la manufactura, reduciendo su incidencia en el crecimiento económico.

Estos antecedentes hacen suponer que el efecto de las exportaciones mineras metálicas en los últimos años sería mayor que el efecto que pudo generar la producción industrial. Sin embargo, esta sería una hipótesis.

Por lo tanto, el presente trabajo tiene por objetivo principal, determinar cuál de las variables mencionadas (exportaciones mineras metálicas o la producción industrial) ha tenido mayor incidencia sobre el crecimiento económico en el Perú en el periodo 1994-2016. Los objetivos específicos son: 1) Determinar la existencia de una relación de equilibrio en el largo plazo entre las exportaciones mineras metálicas, la producción industrial y el crecimiento económico, para hallar una sincronización en el tiempo que refleje una relación confiable y determinante; 2) Determinar la direccionalidad de la relación causal entre las exportaciones mineras metálicas, la producción industrial y el crecimiento económico.
La presente investigación contribuye a esclarecer cuál ha sido el efecto determinante que ha impulsado al crecimiento económico del Perú a partir de su Constitución Política de $1993^{1}$, enfocándose en la relación entre las exportaciones mineras metálicas y producción industrial. Asimismo, en el Perú no hay estudios que comparen específicamente estos efectos utilizando en su metodología las pruebas de cointegración de Johansen y Causalidad de Granger.

\section{Materiales y métodos}

\section{Datos y variables}

En esta investigación, se usaron datos de series de tiempo anuales ${ }^{2}$ para evaluar las relaciones a largo plazo entre las variables. El crecimiento económico será representado mediante el producto bruto interno real (PBI), dando la posibilidad de comparar la producción real en tiempos diferentes. El sector minero está reflejado mediante los datos anuales de valores FOB de la exportación de minerales metálicos (cobre, zinc, oro, plata, estaño, hierro plomo y molibdeno), los cuales se tomaron como una sola variable (XMIN) y el sector industrial mediante el Producto Bruto Interno Manufacturero (PBIMAN).

PBI: Producto bruto interno real (millones S/ 2007). PBIMAN: Producto Bruto Interno manufacturero (millones S/ 2007). XMIN: Valores FOB de las exportaciones mineras (millones US\$)

La data se tomó de las series estadísticas del BCRP, desde 1994 hasta el 2016 (se escoge este periodo debido a que a partir de 1993 rige la nueva Constitución Política del Perú, que permitió el mayor ingreso de inversiones a los diferentes sectores de la

\footnotetext{
${ }^{1}$ La Constitución política de 1993 permitió el mayor ingreso de inversiones a los diferentes sectores de la economía nacional.

${ }^{2}$ Por convención, las series de tiempo anuales no pueden contener estacionalidad (Centro de Investigación y Desarrollo del Instituto Nacional de Estadística e Informática, 2002).
} 
economía nacional), se usaron precios del 2007, debido al fuerte aumento en el peso del sector minero, pues fue un año de precios de los metales excepcionalmente altos. Para desarrollar la metodología planetada, los datos fueron procesados en el programa econométrico EViews 9.

\section{Metodología}

El procedimiento econométrico utilizado en la presente investigación se respalda en estudios hechos por Mata (2003), Londoño (2005), Sahoo (2014), Novales (2014), Banegas (2015) y Mayurí (2015). El esquema planteo un análisis de raíz unitaria, para probar la estacionariedad de las series. Después se estimó un modelo VAR con rezagos óptimos y en base a ello se determinó el rango de cointegración. Posteriormente, se estimó un modelo VEC, a partir del cual se hallaron las ecuaciones de cointegración que guardan la relación de equilibrio en el largo plazo; por último, la relación causal, que reforzó el análisis en torno a la relación de las variables.

\section{Modelo de vectores autorregresivos (VAR)}

Es un modelo de ecuaciones simultaneas que describe las relaciones dinámicas de las variables (Novales, 2014). El modelo VAR produce estimadores eficientes y consistentes, siempre y cuando los términos de error sean de ruido blanco, es decir, no esten correlacionados. La motivación de usar este modelo es mejorar la predicción de las series temporales y captar dependencias dinámicas que pudiera haber entre las variables. Además, evita la dificultad de identificar a las varibles como exógenas (Novales, 2014).

En un vector sin restriciones, el modelo de vectores autoregresivo (VAR) podría expresarse de la siguiente manera.

$$
\mathbf{Y} t=m+\sum_{(\mathbf{i}=\mathbf{1})}^{\mathbf{P}} \mathbf{A}_{\mathbf{i}} \mathbf{Y}_{(\mathrm{t}-\mathrm{i})}+\boldsymbol{u}_{t}
$$

Donde:

$\mathbf{Y} t$ : Vector de variables no estacionarias

$\mathbf{Y}_{(\mathrm{t}-\mathrm{i})}$ : Vector de variables no estacionarias retardado

$\mathbf{A}_{\text {I }}:$ Matriz de coeficientes

$m$ : Constante

$P \quad$ : Longitud de retardos

$\boldsymbol{u}_{\boldsymbol{t}}$ : Término de error

\section{Estimación}

Primero se realizó una prueba de raíz unitaria aumentada Dickey-Fuller (ADF), la cual garantiza que los términos de error no estén correlacionados, probando el orden de integración, es decir, la cantidad de veces que hay que diferenciar una serie hasta convertirla en estacionaria (Mahadeva y Robinson, 2004).

Según Engler y Nahuelhual (2003), una serie es estacionaria cuando su media y varianza son estables; y es no estacionaria cuando no evidencia una tendencia a regresar a su media y su varianza tiende a crecer en el tiempo. Cabe resaltar que cuando se incluye series no estacionarias en un modelo, abre la posibilidad de que cause la existencia de regresiones espurias (Mahadeva y Robinson, 2009).

La prueba Aumentada Dickey- Fuller (ADF) consiste en determinar la presencia de raíces unitarias, es decir, series no estacionarias (Dickey y Fuller, 1981).

$\Delta Y_{t}=m+b t+\theta Y_{(t-1)}+\sum_{(i=l)}^{(p-l)} B i \Delta Y_{(t-i)}+u_{t}$

Donde:

$\Delta \mathrm{Y}_{\mathrm{t}}$ : Vector diferenciado de la variable no estacionaria

$m$ : Término Constante

bt: Término de Tendencia 
$\mathrm{B}_{\mathrm{i}}$ : Matriz de parametros para

$\Theta:$ Parametro de la variable retardada

$P$ : Longitud de retardos

$u_{t}$ : Término de error

Primero se graficaron las series PBI, PBIMAN y XMIN para observar si existe una tendencia determinista, lo cual implicaría la presencia de raíces unitarias. Posteriormente, se aplicó la prueba formal de raíces unitarias ADF, tanto en niveles
El rezago óptimo responderá al criterio de información que reporte el valor mínimo en el rango de rezagos considerados.

Una vez conocidos el número de rezagos óptimos, se procedió a estimar el modelo VAR, el cual describió las relaciones dinámicas de las variables PBI, XMIN y PBIMAN, siendo todas consideradas como endógenas y representadas de la siguiente manera:

$$
Y_{t}=(P B I, X M I N, P B I M A N)
$$
como en primeras diferencias, para $P B I_{t}=m_{l}+\sum_{i=1}^{P} \alpha_{t} P B I t-i+\sum_{j=1}^{P} \beta_{j} P B I M A N_{t-j}+\sum_{z=1}^{P} \omega_{z} X M I N t-z+u_{I t}$ determinar el orden de integración de las variables a incluirse en el modelo PBIMAN $_{t}=m_{2}+\sum_{i=l}^{P} \alpha_{i} P B I M A N_{t-i}+\sum_{j=1}^{P} \beta_{j} P B I_{t-j}+\sum_{z=1}^{P} \omega_{z} X M I N_{t-z}+u_{2 t}$ VAR

Se reportó el estadístico ADF a fin $X M I N_{t}=m_{3}+\sum_{i=1}^{P} \alpha_{t} X M I N_{t-i}+\sum_{j=1}^{P} \beta_{j} P B I M A N_{t-j}+\sum_{z=1}^{P} \omega_{z} P B I_{t-z} u_{3 t}$ probar la hipotésis nula de que el parámetro $\Theta$ de la variable retardada Luego se buscó evaluar que los residuos del modelo es cero.

Se aplicó el test estadístico ADF a las series de PBI, PBIMAN y XMIN en niveles, donde el parámetro de interés es $\Theta$. Por el contrario, si presentan raíces unitarias (series no estacionarias), entonces se deberá determinar el orden de integración. La serie será integrada de primer orden I(1) si requiere que se le realice la primera diferencia; de segundo orden I(2) si requiere la segunda diferencia y así sucesivamente hasta que el estadístico ADF sea lo suficientemente negativo para cumplir las propiedades de estacionariedad.

Las series que presenten raíces unitarias (series no estacionarias) y un mismo orden de integración, se colocaran en un vector autorregresivo (VAR) a partir del cual se podrá determinar la existencia de vectores de cointegración (r) (Perdomo, 2002).

Posteriormente, se debe seleccionar el rezago óptimo en el modelo VAR. Para seleccionar la longitud de rezagos, se utilizó el estadístico de relación de probabilidad (LR) y los criterios de información de Akaike (ACI), Schwarz (SC), Hanna Quinn (HQ) y predicción final de error (FPE) (Mata, 2003).

VAR estimado, cuente con las condiciones necesarias para dar validez al modelo ${ }^{3}$, es decir, que no presenten problemas de normalidad, heterocedasticidad y autocorrelación.

\section{Prueba de cointegración de Johansen}

La prueba de cointegración de Johansen (1988) es usada para probar la relación a largo plazo entre las variables. Las series están cointegradas si se mueven conjuntamente a lo largo del tiempo y son estacionarias, y aún si la serie individual tiene una tendencia estocástica, es decir, si fuera no estacionaria. Johansen \& Juselius (1990) sostiene que la cointegración ofrece una sincronización en el tiempo que refleja una relación confiable entre las variables.

La cointegración refleja la presencia de un equilibrio a largo plazo hacia el

\footnotetext{
${ }^{3} \mathrm{Al}$ ser series de tiempo que comparten una tendencia en común, puede existir multicolinealidad, sin embargo, esta no viola los supuestos básicos de la regresión en el modelo VAR, ya que, a pesar de su existencia, las estimaciones serán consistentes e insesgadas y sus errores estándar se estimarán en la forma correcta (Gujarati y Porter, 2010).
} 
cual converge el sistema económico en el tiempo (Mata, 2003). Asimismo, Johansen \& Juselius (1990) asume que en el modelo todas las variables son endógenas y que las series son integradas del mismo orden I(d). Según Sahoo et al. (2014), existirá una relación de largo plazo entre las variables si la combinación lineal de variables no estacionarias de orden I(1) producen residuos que son estacionarios de orden $\mathrm{I}(0)$ (ruido blanco).

\section{Estimación}

Para probar la existencia del número de vectores de cointegración (r) entre las variables, se aplicó dos estadísticas basadas en la prueba de máxima verosimilitud con el fin de determinar el rango de cointegración del sistema. Asimismo, cabe resaltar que ambas pruebas deberán reflejar los mismos resultados para dar una conclusión generalizada.

\section{a. máximo valor propio( $(\lambda \max )$}

$$
\lambda_{\max }=-\mathrm{T} \ln (1-\lambda \hat{i})
$$

Donde $\lambda \hat{i}$ son los valores propios y $\mathrm{T}$ es el número de observaciones menos el número de rezagos.

\section{b. la prueba de traza (Atrace)}

$\lambda_{\text {trace }}=\sum_{\mathrm{i}=\mathrm{r}+1}^{\mathrm{N}} \ln \left(1-\lambda_{\mathrm{i}}\right)$

Donde los son los valores propios, $\mathrm{N}$ es la cantidad de variables endógenas.

Según Londoño (2005), si se tiene $\mathrm{N}$ variables endógenas y estas son no estacionarias, pueden presentarse de cero hasta N-1 relaciones de cointegración linealmente independientes. Las ecuaciones 6 y 7 deberán coincidir en el rango de cointegración.

Si las variables PBI, PBIMAN y XMIN no cointegran, se mantendrá el modelo VAR. Caso contrario, si existiera cointegración en el modelo, será necesario estimar un modelo de vectores de corrección de error (VEC), el cual tomará en cuenta el rango de cointegración hallado (Perdomo, 2002).

\section{Modelo de vectores de corrección de error (VEC)}

El modelo de Vectores de Corrección de Error (VEC) es un tipo de modelo VAR para variables no estacionarias que están cointegradas. En el modelo VEC, todas las variables son estacionarias en primeras diferencias y están expresadas como función lineal de valores pasados de sí misma (rezagos), valores pasados del resto de variables del modelo y de los vectores de cointegración (Engler y Nahuelhual, 2003). El Modelo VEC se expresa de la siguiente manera:

$$
\begin{aligned}
& \Delta Y_{t}=m+\lambda E C q+\sum_{i=1}^{P-1} R_{i} \Delta Y_{t-i}+u_{t} \\
& \quad \mathrm{Ri}=-(\mathrm{I}-\mathrm{A} 1-\mathrm{A} 2-\cdots-\mathrm{Ap}) ;(\mathrm{i}=1,2, \cdots, \mathrm{p}-1)
\end{aligned}
$$

Donde:

$\Delta Y_{t}$ : Vector diferenciado de variables endógenas no estacionarias

$\Delta Y_{t-i}$ : Vector diferenciado retardado de variables endógenas no estacionarias

$E C q$ : Término de correción del error $\mathrm{y}$ ecuación de cointegración

$R_{i}$ : Parámetros dinámicos de las variables retardadas

$\lambda \mathrm{i}$ : Velocidad del parámetro de ajuste al equilibrio

$m$ : Constante

$u_{t}$ : Término de error no correlacionado

\section{Estimación}

De hallarse vectores de cointegración en el modelo VAR, entonces se estimará el 
modelo VEC, determinando la relación de las variables PBI, PBIMAN y XMIN en términos de primeras diferencias. Debido a que se aplicó la primera diferencia, se especificará un rezago menos con respecto al número de rezagos óptimo. estimar $y$. Sin embargo, cabe resaltar que el hecho de que $x$ cause a $y$, no implica que $y$ sea el efecto de $x$, pues intervienen diversos factores que son ajenos a $x$.

\section{Estimación}

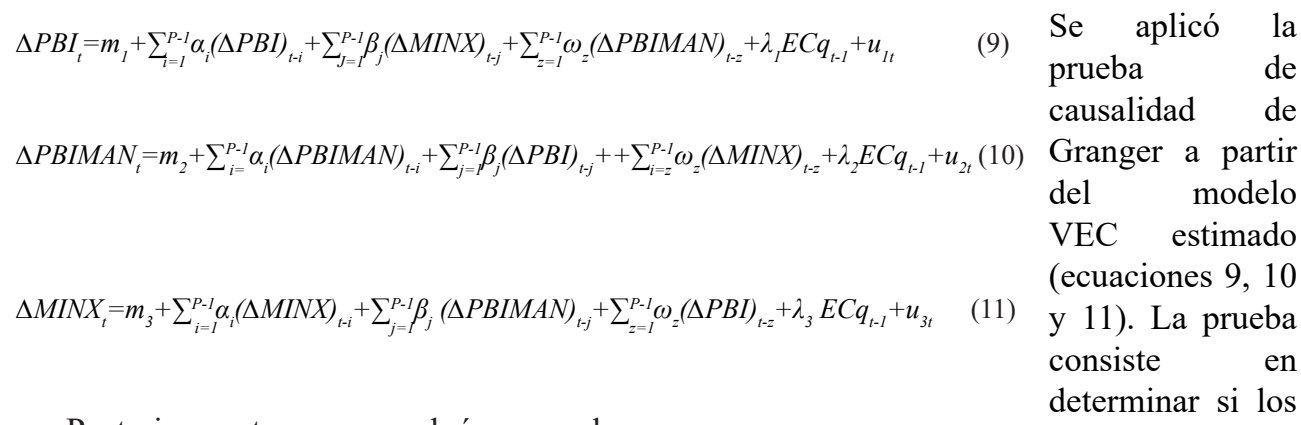

Posteriormente, se evaluó que los residuos del modelo VEC estimado, cuenten con las condiciones necesarias para dar validez al modelo, es decir, que no presenten problemas de normalidad, heterocedasticidad y autocorrelación.

Las ecuaciones de cointegración serán dadas por el modelo VEC y determinarán la relación de equilibrio en el largo plazo entre el PBI, PBIMAN Y XMIN.

Según Novales (2014), la determinación de la convergencia del sistema dependerá del coeficiente $\lambda_{\mathrm{i}}$ de la ecuación de cointegración $E C q$, pues medirá la velocidad de ajuste de las variables PBI, PBIMAN y XMIN hacia el equilibrio en el largo plazo, y esta debe ser negativa, ya que la corrección tendría que apuntar a una dirección contraria para asegurar que el equilibrio se mantenga y se corrija.

\section{Prueba de causalidad Granger}

Esta prueba es utilizada para determinar la relación causal entre las variables. Según Granger (1969), existe una relación causal si el valor pasado y presente de una variable ayuda a predecir la otra variable. Es decir, la variable $y$ es causada por $x$, si $x$ contribuye a parametros de las variables retardadas son estadisticamente diferentes de cero.

Se reportó el estadístico $\mathrm{F}$ a fin de probar Las relaciones de causalidad halladas entre las variables de las ecuaciones 9, 10 y 11 del modelo VEC, reforzarán la relación de equilibrio en el largo plazo, determinando el impulso de una variable a otra a lo largo de su relación en el tiempo.

\section{Resultados y discusión}

\section{Estimación del modelo del vector autorregresivo (VAR)}

En la Figura 1, se puede observar que las series presentan tendencia determinista creciente, es decir, son series no estacionarias, lo cual implicaría la presencia de raíz unitaria.

Para verificar si las series de tiempo son estacionarias, se realizó la prueba Aumentada Dickey - Fuller (ADF), tanto en niveles como en primeras diferencias para determinar el orden de integración de las variables a incluirse en el modelo VAR. Debido a que las series presentan una tendencia, se incluyó en la prueba de ecuación la constante y tendencia. En las Tablas 1 y 2 se muestran los resultados. 


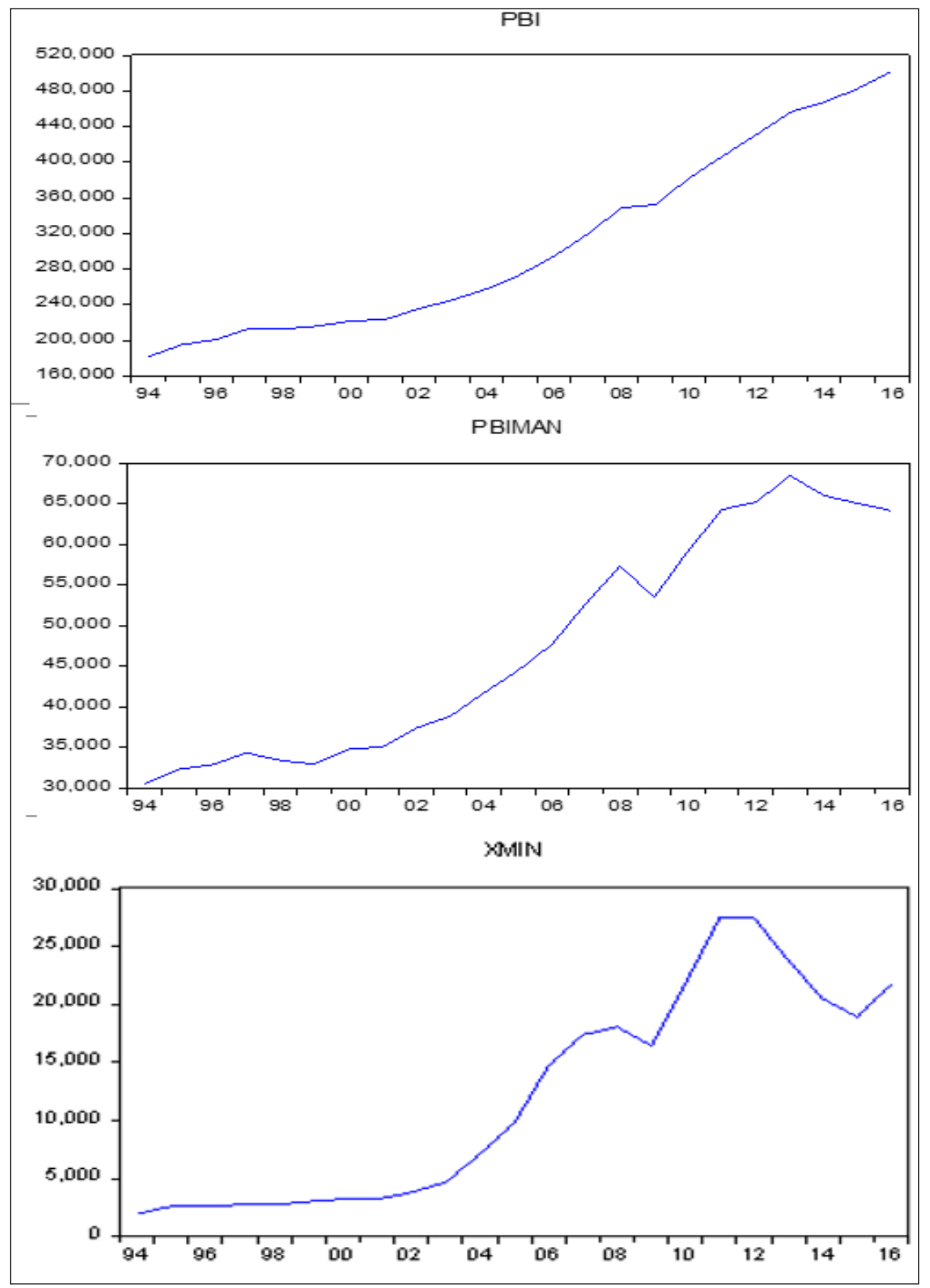

Figura 1. Representación lineal de las series de tiempo 
Tabla 1. Prueba ADF aplicada a las variables en niveles

\begin{tabular}{clrrr}
\hline Variable & \multicolumn{1}{c}{ Modelo } & t-statistic & \multicolumn{1}{c}{$5 \%$} & \multicolumn{1}{c}{ Prob } \\
\hline \multirow{2}{*}{ PBI } & constante & 2,701587 & $-3,004861$ & 1 \\
& constante y tendencia & $-1,167693$ & $-3,632896$ & 0,8925 \\
\multirow{2}{*}{ PBIMAN } & constante & $-0,449685$ & $-3,004861$ & 0,8836 \\
& constante y tendencia & $-1,62708$ & $-3,632896$ & 0,7485 \\
\multirow{2}{*}{ XMIN } & constante & $-0,458719$ & $-3,020686$ & 0,8803 \\
& constante y tendencia & $-3,273127$ & $-3,644963$ & 0,098 \\
\hline
\end{tabular}

Tabla 2. Prueba ADF aplicada a las variables en primeras diferencias

\begin{tabular}{clrrr}
\hline Variable & \multicolumn{1}{c}{ Modelo } & t-statistic & \multicolumn{1}{c}{$5 \%$} & \multicolumn{1}{c}{ Prob } \\
\hline \multirow{2}{*}{ PBI } & constante & $-2,835661$ & $-3,012363$ & 0,0703 \\
& constante y tendencia & $-4,156912$ & $-3,644963$ & 0,0186 \\
\multirow{2}{*}{ PBIMAN } & constante & $-4,081955$ & $-3,012363$ & 0,0053 \\
& constante y tendencia & $-3,95981$ & $-3,644963$ & 0,0274 \\
\multirow{2}{*}{ XMIN } & constante & $-3,980087$ & $-3,020686$ & 0,0069 \\
& constante y tendencia & $-3,853182$ & $-3,658446$ & 0,0348 \\
\hline
\end{tabular}

En la Tabla 1 se observa que los estadísticos ADF son mayores que los valores críticos al $5 \%$ y que las probabilidades no son significativas $(>0,05)$, por lo tanto, no se rechaza $\mathrm{H}_{0}$ : La serie de PBI, PBIMAN y XMIN son no estacionaria. Entonces, se concluye que las variables en niveles presentan raíz unitaria (series no estacionarias).

Mientras que en la Tabla 2 se observa que los estadísticos ADF son menores que los valores críticos al $5 \%{ }^{4}$ y que las probabilidades son significativas $(<0,05)$, por lo tanto, se rechaza $\mathrm{H}_{0}$ : La serie de PBI, PBIMAN y XMIN son no estacionarias. Entonces, se concluye que las variables en primera diferencia no presentan raíz unitaria (series estacionarias). En consecuencia, las series son de primer orden I(1).

La Tabla 3 presenta los resultados de la estimación del número de rezagos óptimos. En un rango de rezagos, considerando el número de variables del modelo, el valor

\footnotetext{
${ }^{4} \mathrm{El} \mathrm{PBI}$ en primeras diferencias no es significativo al $5 \%$, ya que el estadístico ADF es mayor al valor crítico. En consecuencia, se tomará un valor crítico al 10\%, siendo este $-2,646119$.
}

mínimo de los criterios de información AIC, HQ, SC y FPE, responden a tres rezagos $(\mathrm{P}=3)$ como longitud óptima para el modelo. Asimismo, la probabilidad del estadístico LR es significativa con 3 rezagos.

En la Tabla 4 se muestra los resultados de las pruebas realizadas a los residuos del modelo VAR. Se encontró que los residuos se encuentran dentro de una distribución normal estándar, que no existe correlación entre ellos y que tienen la misma varianza. En conclusión, se puede apreciar que los residuos no presentan problemas de normalidad, autocorrelación y heterocedasticidad.

\section{Resultados del análisis de cointegración}

Dado que el modelo VAR cumple con todas las pruebas de residuos y las series son estacionarias, se aplicó la Prueba de Cointegración de Johansen. En el contraste de la ecuación, se permitió la tendencia determinística lineal en los datos (Intercepto - no tendencia). Se presenta los resultados de la prueba Máximo Valor Propio en la Tabla 5 y la prueba de Traza en la Tabla 6. 
Tabla 3. Número de rezagos óptimos para el modelo VAR

\begin{tabular}{ccc}
\hline Criterios de información & Valor mínimo & Longitud óptimo (P) \\
\hline Akaike (AIC) & 54,18351 & 3 rezagos \\
Schwarz (SC) & 55,67711 & 3 rezagos \\
Hannan-Quinn (HQ) & 54,47508 & 3 rezagos \\
Predicción final de error (FPE) & $9,18 \mathrm{E}+19$ & 3 rezagos \\
\hline
\end{tabular}

Tabla 4. Resultado del análisis de los residuos del modelo VAR

\begin{tabular}{ccl}
\hline Tipo de Prueba & Probalidad & \multicolumn{1}{c}{ Conclusión } \\
\hline Test de Jarque - Bera & 0,1872 & $\begin{array}{l}\text { La Probabilidad del test es mayor a 0,05 (5\%), por lo tanto, } \\
\text { no se rechaza Ho: Residuos son normales. Es decir, no existe } \\
\text { problemas de normalidad. }\end{array}$ \\
$\begin{array}{c}\text { Test de multiplicador } \\
\text { de Lagrange (LM) }\end{array} \quad 0,2038$ & $\begin{array}{l}\text { La probabilidad del test es mayor a 0,05 (5\%), por lo tanto, } \\
\text { no sechaza Ho: Ausencia de autocorrelación hasta el } \\
\text { retardo de orden h. Es decir, los residuos del modelo no } \\
\text { presentan problemas de autocorrelación. } \\
\text { La probabilidad del test es mayor a 0,05 (5\%), por lo tanto, } \\
\text { no se rechaza Ho: Residuos homocedásticos. Es decir, los } \\
\text { residuos del modelo son homocedásticos. }\end{array}$ \\
$\begin{array}{c}\text { Test de White sin } \\
\text { términos cruzados }\end{array}$ & 0,3296
\end{tabular}

Tabla 5. Resultados de la Prueba Máximo Valor Propio

\begin{tabular}{cccc}
\hline $\begin{array}{c}\text { Rango de } \\
\text { cointegración }\end{array}$ & $\begin{array}{c}\text { Estadístico de Máximo } \\
\text { valor propio }\end{array}$ & $\begin{array}{c}\text { Valor crítico } \\
0.05\end{array}$ & Prob \\
\cline { 1 - 3 }$r=0$ & 28,27916 & 21,13162 & 0,0042 \\
$r=1$ & 21,01039 & 14,2646 & 0,0037 \\
$r=2$ & 2,651599 & 3,841466 & 0,1034 \\
\hline
\end{tabular}

Tabla 6. Resultados de la Prueba Traza

\begin{tabular}{cccc}
\hline $\begin{array}{c}\text { Rango de } \\
\text { cointegración }\end{array}$ & Estadístico de traza & $\begin{array}{c}\text { Valor crítico } \\
0.05\end{array}$ & Prob \\
\hline $\mathrm{r}=0$ & 51,94114 & 29,79707 & 0 \\
$\mathrm{r} \leq 1$ & 23,66199 & 15,49471 & 0,0024 \\
$\mathrm{r} \leq 2$ & 2,651599 & 3,841466 & 0,1034 \\
\hline
\end{tabular}

Al obtener 28,27916 > 21,13162, existe al menos una relación de equilibrio en el largo plazo. Mientras que 21,01039 > 14,26460, existe más de una relación de equilibrio en el largo plazo. Asimismo 2,651599<3,841466, existe hasta dos relaciones de equilibrio en el largo plazo.

La prueba de Máximo Valor Propio señala que existen 2 vectores de cointegración $(\mathrm{r}=2)$, es decir, dos relaciones de equilibrio en el largo plazo en el modelo.
Al obtener 51,94114 > 29,79707, existe al menos una relación de equilibrio en el largo plazo. Mientras que 23,66199 > 15,49471 existe más de una relación de equilibrio en el largo plazo. Asimismo 2,651599 < 3,841466 existe hasta dos relaciones de equilibrio en el largo plazo.

La prueba de Traza señala que existen 2 vectores de cointegración ( $\mathrm{r}=2$ ), es decir, dos relaciones de equilibrio en el largo plazo en el modelo. 
En conclusión, existe dos vectores de cointegración $(\mathrm{r}=2)$ en el modelo, es decir, existen dos relaciones de equilibrio en el largo plazo entre las variables.

\section{Modelo del vector de corrección del error (VEC)}

La existencia de vectores de cointegración en el modelo VAR implica que existe una relación estable de equilibrio a largo plazo entre las variables. Sin embargo, en el corto plazo puede haber desequilibrio. En consecuencia, se aplicó el modelo VEC para corregir este desequilibrio. Este modelo abarca las relaciones de corto y largo plazo.

Para estimar el modelo VEC, el cual contiene a las ecuaciones de integración, se determinó la relación de las variables PBI, PBIMAN y XMIN en términos de primeras diferencias, tomando en cuenta el rango de cointegración $(\mathrm{r}=2)$ obtenido en el modelo VAR y se especificó un rezago menos con respecto al número de rezago óptimo, porque cuando se aplica primera diferencia, en el modelo se genera un vacío por la pérdida de un rezago. Las ecuaciones de cointegración de largo plazo son:

$$
\operatorname{ECq}(1): P B I_{t-1}=8,348312 * X M I N_{t-1}+208194,9
$$

$E C q(2): P_{B M A N}=1,230370 * X M I N_{t-1}+32953,56$
Existe dos ecuaciones de cointegración de largo plazo: $E C q(1)$ representa la existencia de una relación de equilibrio en el largo plazo entre las exportaciones minera metálicas y el crecimiento económico del país. $E C q(2)$ representa la existencia de una relación de equilibrio en el largo plazo entre las exportaciones minera metálicas y la producción manufacturera.

En la Tabla 7 se muestra los resultados de las pruebas realizadas a los residuos del modelo VEC. Dichos resultados arrojaron que los residuos se encuentran dentro de una distribución normal estándar, que no existe correlación entre ellos y que tienen la misma varianza. En conclusión, se puede apreciar que los residuos no presentan problemas de normalidad, autocorrelación y heterocedasticidad.

Asimismo, se presenta el modelo de corrección de errores estimado, donde los efectos son las diferencias de las variables que los va corrigiendo desde el corto plazo a medida que pasa el tiempo, tal de que, en un escenario de largo plazo, se hayan corregido los errores de estimación que pudiera haber arrastrado estas diferencias.

A continuación se presenta el modelo de Corrección de errores (VEC) estimado:

Tabla 7. Resultado del análisis de los residuos del modelo VEC

\begin{tabular}{ccl}
\hline Tipo de Prueba & Probalidad & \multicolumn{1}{c}{ Conclusión } \\
\hline Test de Jarque - Bera & 0,3595 & $\begin{array}{l}\text { La Probabilidad del test es mayor a 0,05 (5\%), por lo tanto, } \\
\text { no se rechaza H0: Residuos son normales. Es decir, no existe } \\
\text { problemas de normalidad. }\end{array}$ \\
$\begin{array}{c}\text { Test de multiplicador de } \\
\text { Lagrange (LM) }\end{array} \quad 0,7276$ & $\begin{array}{l}\text { La probabilidad del test es mayor a 0,05 (5\%), por lo tanto, no } \\
\text { se rechaza H0: Ausencia de autocorrelación hasta el retardo } \\
\text { de orden h. Es decir, los residuos del modelo no presentan } \\
\text { problemas de autocorrelación. } \\
\text { La probabilidad del test es mayor a 0,05 (5\%), por lo tanto, } \\
\text { no se rechaza H0: Residuos homocedásticos. Es decir, los } \\
\text { residuos del modelo son homocedásticos }\end{array}$ \\
$\begin{array}{c}\text { Test de White sin } \\
\text { términos cruzados }\end{array}$ & 0,2223 \\
\hline
\end{tabular}


$\Delta P B I_{t}=-0,2984 E C q(1)+4,6586 E C q(2)+0,1389 \Delta P B I_{t-1}+0,5624 \Delta P B I_{t-2}-5,3080 \Delta P B I M A N_{t-1}-4,0672 \Delta P B I M A N_{t}$ ${ }_{2}+4,2002 \Delta X M I N_{t-1}+4,0421 \Delta X M I N_{t-2}+13779,94$

$\triangle P B I M A N_{t}=-0,1532 E C q(1)+1,6150 E C q(2)+0,0525 \Delta P B I_{t-1}+0,0233 \Delta P B I_{t-2}-1,9375 \Delta P B I M A N_{t-1}-1,1883 \triangle P B I M A N_{t}$ ${ }_{2}+1,1531 \Delta{ }^{t} \operatorname{MIN}_{t-1}+1,0322 \Delta X M I N_{t-2}+3860,391$

$\Delta \mathrm{XMIN}_{\mathrm{t}}=-0,2469 \mathrm{ECq}(1)+3,002 \mathrm{ECq}(2)-0,0808 \Delta \mathrm{PBI}_{\mathrm{t}-1}+0,0292 \Delta \mathrm{PBI}_{\mathrm{t}-2}-2,1032 \Delta \mathrm{PBIMAN}_{\mathrm{t}-1}-1,5133 \Delta \mathrm{PBIMAN}_{\mathrm{t}}$ ${ }_{2}+1,6989 \Delta \mathrm{XMIN}_{\mathrm{t}-1}+0,8406 \Delta \mathrm{XMIN}_{\mathrm{t}-2}+5662,585$

Tomando en cuenta las ecuaciones de cointegración $(\mathrm{ECq}) 12$ y 13 que guardan la relación de equilibrio en el largo plazo de PBI-XMIN y PBIMAN-XMIN respectivamente, se interpretó las ecuaciones del modelo VEC.

En la ecuación 14 se observa que el coeficiente de $\mathrm{ECq}(1)$ es negativo, lo cual indica que si el valor de XMIN se encuentra por encima de su equilibrio a largo plazo, entonces el PBI aumentará en el próximo periodo con una velocidad de ajuste de $29,8 \%$, dando una buena señal para que el sistema converja. Esto sugiere la importancia de los minerales metálicos en la economía peruana. No se tomó en cuenta $\mathrm{ECq}(2)$, al no tener relación con $\triangle P B I_{\mathrm{t}}$.

En la ecuación 15 se observa que el coeficiente de $\operatorname{ECq}(2)$ es positivo, lo cual indica que si el valor de XMIN se encuentra por encima de su equilibrio a largo plazo, entonces PBIMAN caerá en el próximo periodo con una velocidad de ajuste de $161,5 \%$, dando una mala señal para que el sistema converja. Esto sugiere que no hay una relación directa inmediata entre las exportaciones mineras metálicas y la producción industrial. No se toma en cuenta $\operatorname{ECq}(1)$, al no tener relación con $\triangle P B I M A N_{t}$.

En la ecuación 16 se observa que el coeficiente de $\mathrm{ECq}(1)$ es negativo, lo cual indica que si el valor de PBI se encuentra por encima de su equilibrio a largo plazo, entonces XMIN aumentará en el próximo periodo con una velocidad de ajuste de $24,7 \%$, dando una buena señal para que el sistema converja. Asimismo, se observa que el coeficiente de $\operatorname{ECq}(2)$ es positivo, lo cual indica que si el valor de PBIMAN se encuentra por encima de su equilibrio a largo plazo, entonces XMIN caerá en el próximo periodo con una velocidad de ajuste de $300 \%$, dando una mala señal para que el sistema converja, lo cual puede deberse a inestabilidades en el modelo o puede haber influencias de otras variables que no han sido consideras.

La relación de equilibrio en el largo plazo entre el PBI y XMIN, mostrada en la ecuación 12 , indica la estrecha relación que persiste en el tiempo y reafirma la incidencia que han tenido los minerales metálicos en el crecimiento de la economía peruana. Además, no se encontró un equilibrio en el largo plazo entre el PBI y PBIMAN, lo cual sugiere que la producción manufacturera no ha sido crucial para determinar el crecimiento económico.

Discutiendo los resultados obtenidos, se observa la concordancia con Sahoo et al. (2014), Shafiullah et al. (2016) y Ee (2016), respecto a la relación de equilibrio de largo plazo entre el crecimiento económico de un país y sus exportaciones mineras. En el plano nacional, Bobadilla (2016) coincide con este estudio al demostrar lo determinantes que son las exportaciones mineras metálicas para la economía peruana a través una relación de equilibrio en el largo plazo, lo cual contradice a Jiménez (2017), ya que en los resultados obtenidos no se halló que la producción manufacturera haya sido crucial para el crecimiento económico del Perú. Asimismo, Díaz y Torres (2016) observaron la existencia de una relación entre el crecimiento económico y las exportaciones 
mineras a través de una regresión lineal, sin embargo, en la presente investigación se optó por hallar la relación de equilibrio de largo plazo, para reflejar el estrecho vínculo a través del tiempo, sin tomar el riesgo de resultados espurios.

Basado en un modelo extractivista, el Perú ha venido creciendo económicamente, teniendo como factor determinante a las exportaciones mineras metálicas, las cuales se han desarrollado en un contexto de altos precios internacionales. Sin embargo, esto podría generar cierta vulnerabilidad ante una caída de precios de los commodities. Al respecto, el BCRP ha mantenido una política fiscal y monetaria que le ha permitido soportar las crisis internacionales que se han presentado (Barco y Castillo, 2009).

\section{Resultados de la causalidad de Granger}

En la Tabla 8 se muestran los resultados de la prueba de causalidad de Granger. Se encontró que existe una causalidad bidireccional exportaciones mineras metálicas y la producción manufacturera, y que ambos impulsaron al crecimiento económico del Perú en el periodo 1994-2016.

Los resultados sugieren que las exportaciones mineras metálicas impulsaron el crecimiento del país en el periodo 19942016. Esta relación causal respalda y fortalece la relación de equilibrio a largo plazo, lo que refleja la incidencia que tuvo la minería metálica para lograr el crecimiento económico del Perú. Asimismo, los resultados señalan que la producción manufacturera impulsa el crecimiento económico, sin embargo, la no existencia de una relación de equilibrio en el largo plazo, desestima la incidencia de la producción manufacturera en el crecimiento económico del Perú. Asimismo, los resultados sugieren que existió una relación de retroalimentación entre la producción manufacturera y las exportaciones de minerales metálicos.
Tabla 8. Resultados Causalidad de Granger

\begin{tabular}{cl}
\hline Probabilidad & \multicolumn{1}{c}{ Conclusión } \\
\hline \multirow{5}{*}{0,0304} & $\begin{array}{l}\text { La probabilidad del test es } \\
\text { menor a 0,05 (5\%), por lo tanto, } \\
\text { se rechaza H0. Es decir, existe } \\
\text { una relación de causalidad desde } \\
\text { el PBIMAN hacia el PBI. } \\
\text { La probabilidad del test es } \\
\text { menor a 0,05 (5\%), por lo tanto, } \\
\text { se rechaza H0. Es decir, existe } \\
\text { una relación de causalidad desde } \\
\text { XMIN hacia el PBI. } \\
\text { La probabilidad del test es mayor } \\
\text { a 0,05 (5\%), por lo tanto, no se } \\
\text { rechaza H0. Es decir, no existe } \\
\text { una relación causalidad desde el } \\
\text { PBI hacia el PBIMAN. } \\
\text { La probabilidad del test es } \\
\text { menor a 0,05 (5\%), por lo tanto, } \\
\text { se rechaza H0. Es decir, existe } \\
\text { una relación de causalidad desde } \\
\text { XMIN hacia el PBIMAN. } \\
\text { La probabilidad del test es mayor } \\
\text { a 0,05 (5\%), por lo tanto, no se } \\
\text { rechaza H0. Es decir, no existe } \\
\text { una relación causalidad desde el } \\
\text { PBI hacia XMIN. } \\
\text { La probabilidad del test es } \\
\text { menor a 0,05 (5\%), por lo tanto, } \\
\text { se rechaza H0. Es decir, existe } \\
\text { una relación de causalidad desde } \\
\text { PBIMAN hacia XMIN. }\end{array}$ \\
\hline 0,5228
\end{tabular}

Discutiendo los resultados de la relación causal, se observa una concordancia con Shafiullah et al. (2016) y Ee (2016) con respecto al hecho de que las exportaciones mineras impulsan el crecimiento económico de los países. Sin embargo, no hay una concordancia con el estudio de Sahoo et al. (2014) realizado en la India, donde el crecimiento económico y la producción industrial impulsan las exportaciones mineras, lo cual es un resultado diametralmente opuesto a los obtenidos en esta investigación. Asimismo, coincide parcialmente con Jiménez (2017) al decir que la producción manufacturera impulsa al crecimiento económico, sin embargo, eso no lo vuelve determinante ya que no existió una relación de equilibrio a largo 
plazo. Probablemente esto se deba a que la concentración de inversión en el sector minero haya afectado al sector industrial reduciendo su competitividad, afectando su incidencia sobre el crecimiento económico Alarco (2011).

Así como el sector minero demanda productos metálicos, maquinaria y equipos para la extracción de los minerales, lo cual permitiría que el sector manufacturero se desarrolle y pueda abastecer a otros sectores productivos, y la entrada de divisas debido a las exportaciones mineras metálicas podrían beneficiar al sector manufacturero a través de bienes de capital y actualizando su tecnología; también es posible que la orientación de inversiones al sector minero debido a una rentabilidad más alta, afecte al sector manufacturero reduciendo su incidencia en el crecimiento económico. Lo cual indica que la retroalimentación entre ambos sectores podría tener implicancias tanto positivas como negativas en la economía peruana.

\section{Conclusiones}

Los resultados sugieren que las exportaciones mineras metálicas han sido determinantes para el crecimiento económico del Perú en el periodo 1994-2016. A pesar que las divisas generadas por la exportación de minerales metálicos ayudan en gran medida a lograr una mayor producción manufacturera, la orientación de inversiones hacia el sector minero pudieron haber ocasionado que el sector manufacturero no se expanda a un ritmo que le permita liderar el crecimiento económico.

Los resultados sugieren que el crecimiento económico del Perú tuvo una relación de equilibrio en el largo plazo con las exportaciones mineras metálicas, mas no con la producción manufacturera. Con una velocidad de ajuste de $24.7 \%$ hacia el equilibrio en el largo plazo, se indica la mayor relevancia de las exportaciones mineras metálicas sobre el crecimiento económico peruano en el periodo 19942016.

Los resultados sugieren que existió una relación de causalidad de las exportaciones mineras metálicas hacia el PBI, es decir, las exportaciones metálicas impulsaron el crecimiento económico del Perú. Esto respalda el equilibrio a largo plazo entre la minería metálica y el crecimiento económico peruano. Asimismo, se evidenció una causalidad de la producción industrial hacia el PBI, sin embargo, no es determinante, ya que no guardan una relación de equilibrio en el largo plazo.

\section{Literatura citada}

Alarco, G. 2011. Exportaciones, tipo de cambio y enfermedad holandesa: el caso peruano. Investigación económica, 70(275): 115-143.

Banegas, R. 2015. Metodología de Cointegración de Johansen y Joselious. Consultado el 14 de diciembre del 2018. Disponible en http://webcache.googleusercontent. com/search?q=cache:yMuwJ8De0a AJ:sac3a018d40842d43.jimcontent. com/download/version/1447268682/ module/10764809760/name/Metodo $\underline{\log \% 25 \mathrm{C} 3 \% 25 \mathrm{ADa} \% 2520 \mathrm{de} \% 2520}$ Cointegraci\%25C3\%25B3n\%2520d e\%2520Johansen\%2520y\%2520Jous elios\%2520(1990) $+\& \mathrm{~cd}=1 \& \mathrm{hl}=\mathrm{es} \& \mathrm{c}$ $\underline{\mathrm{t}=\mathrm{clnk} \& \mathrm{gl}=\mathrm{pe}}$

Barco, D.; Castillo, P. 2009. Crisis financieras y manejo de reservas en el Perú. Banco Central de Reserva del Perú. Disponible en http://www.bcrp. gob.pe/docs/Publicaciones/RevistaEstudios-Economicos/17/EstudiosEconomicos-17-3.pdf

Bobadilla, H. 2016. Recursos minerales: Maldición o bendición para el 
crecimiento de la economía peruana: 1991T1-2015T2. Tesis Econ. Piura, Perú. UDEP. 117 p.

Centro de Investigación y Desarrollo del Instituto Nacional de Estadística e Informática. 2002. Desestacionalización de series económicas. Disponible en https://www.inei.gob.pe/media/ MenuRecursivo/publicaciones digitales/Est/Lib0514/Libro.pdf

Díaz, P.; Torres, J. 2016. El impacto de las exportaciones tradicionales en el crecimiento económico peruano para el periodo 1990-2015. Tesis Econ. Lima, Perú. UIGV. 82 p.

Dickey, A.D.; Fuller, W,A. 1981. Likehood ratio statistics for autoregressive time series with a unit root. Econometrica, 49(4): 1057-1072.

Ee, C. 2016. Export-led growth hypothesis: empirical evidence from selected Sub-Saharan African countries. Procedia Economics and finance, 35: 232-240.

Engler, A.; Nahuelhual, L. 2003. Influencia del mercado internacional de lácteos sobre el precio nacional de la leche: un análisis de cointegración. Disponible en https://www.researchgate.net/ publication/28067124_Influencia del mercado internacional de lacteos sobre el precio nacional de la leche un analisis de cointegracion

Granger, C.W.J. 1969. Investigating causal relations by econometric models and cross-spectral methods. Econometrica, 37(3): 424-438.

Gujarati, D.; Porter, D. 2010. Econometría. 5 ed. Mexico, D.F, MX. McGraw-Hill. $921 \mathrm{p}$.

Hausmann, R.; Hwang, J.; Rodrik, D. 2007. What you export matters. Journal of
Economic Growth 12: 1-25.

Instituto Peruano de Economía. 2017. El valor agregado de la minería en el Perú (en línea). IDEM. Disponible en http://www.ipe.org.pe/portal/ wp-content/uploads/2018/01/IPEJunio-2017-El-Valor-Agregado-dela-Miner $\%$ C3\%ADa.pdf

Jiménez, F. 2017. Crecimiento y desindustrialización prematura en Perú 1950-2015, un análisis kaldoriano. Economía, N80: 155 222.

Johansen, S. 1988. Statistical analysis of cointegration vectors. Journal of Economics and Control 12: 231-254.

Johansen, S.; Juselius, K. 1990. Maximum likelihood estimation and inference on cointegration-with applications to the demand for money. Oxford Bulletin of Economics and Statistics, 52(2): 169-210.

Londoño, W. 2005. Modelos de ecuaciones múltiples: modelos VAR $\mathrm{y}$ cointegración. Universidad EAFIT. Disponible en https://core.ac.uk/ download/pdf/47236804.pdf

Mahadeva, L.; Robinson, P. 2004. Unit root testing to help model building. Handbooks in Central Banking, $\mathrm{N}^{\circ} 22$.

Mahadeva, L.; Robinson, P. 2009. Prueba de raíz unitaria para ayudar a la construcción de un modelo. México. D.F. Centro de Estudios Monetarios Latinoamericanos. $3 \mathrm{p}$ (serie de ensayos $\mathrm{N}^{\circ} 76$ ).

Mata, H. 2003. Nociones Elementales de Cointegración Enfoque de Engel Granger. Disponible en http:// webdelprofesor.ula.ve/economia/ hmata/Notas/Engle\%20Granger.pdf

Mayurí, J. 2015. La inversión en infraestructura pública $\mathrm{y}$ el 
crecimiento económico en el Perú, periodo 1950-2013. Tesis Econ. Lima, Perú. UNALM. 67 p.

Novales, A. 2014. Modelos vectoriales autoregresivos (VAR). Universidad Complutense. Disponible en https:// www.ucm.es/data/cont/media/www/ pag-41459/VAR_new.pdf

Perdomo, A. 2002. Inversión pública sectorial y crecimiento económico: una aproximación desde la metodología VAR. Bogotá D. C., CO. 7-29 p. (Serie Archivos de Economía documento 208).

Sahoo, A.; Sahoo, D.; Sahu, N. 2014. Mining export, industrial production and economic growth: A cointegration and causality analysis for India. Resources Policy, 42: 27-34.

Shafiullah, M.; Selvanathan, S.; Naranpanawa, A. 2016. The role of export composition in export-led growth in Australia and its regions. Economic Analysis and Policy. 53: 62-76.

Tang, C.; Lai, Y.; Ozturk, I. 2015. How stable is the export-led growth hypothesis? Evidence from Asia's four little dragons. Economic Modelling, 44: 229-235. 